\title{
Quenching Rate for the Porous Medium Equation with a Singular Boundary Condition
}

\author{
Zhengce Zhang, Yanyan Li \\ College of Science, Xi' an Jiaotong University, Xi' an, China \\ E-mail: zhangzc@mail.xjtu.edu.cn,liyan86911@126.com \\ Received July 3, 2011; revised July 24, 2011; accepted August 1, 2011
}

\begin{abstract}
We study the porous medium equation $u_{t}=\left(u^{m}\right)_{x x}, 0<x<\infty, t>0$ with a singular boundary condition $\left(u^{m}\right)_{x}(0, t)=u^{-\beta}(0, t)$. We prove finite time quenching for the solution at the boundary $x=0$. We also establish the quenching rate and asymptotic behavior on the quenching point.
\end{abstract}

Keywords: Porous Medium Equation, Quenching Profile, Quenching Rate, Singular Boundary Condition

\section{Introduction}

The nonlinear diffusion equation

$$
u_{t}=\Delta\left(u^{m}\right)
$$

with exponent $m>1$, is usually called the porous medium equation, written here PME for short. In the particular case $m=2$, it is called Boussinesq's equation. The PME equation is one of the simplest examples of a nonlinear evolution equation of parabolic type. It appears in the description of different natural phenomena, and its theory and properties depart strongly from the heat equation $u_{t}=\Delta u$, its most famous relative.

There are a number of physical applications where this simple model appears in a natural way, mainly to describe processes involving fluid flow, heat transfer or diffusion. Maybe the best known of them is the description of the flow of an isentropic gas through a porous medium, modelled independently by Leibenzon [1] and Muskat [2] around 1930, where $u$ represents the density of the gas and $m \in[2, \infty)$ is a constant. The most striking manifestation of this nonlinear degeneracy is that in porous medium flow there is a finite speed of propagation of disturbances from rest.

Once the theory for the PME began to be known, a number of applications have been proposed. Some of them concern the fast diffusion equation, the generalized PME and the inhomogeneous versions already commented. There are numerous examples with lower order terms, in the areas of reaction-diffusion, where the PME

\footnotetext{
*Supported by the Fundamental Research Funds for the Central Universities of China.
}

is only responsible for one of the various mechanisms of the equation or system.

In [3], it is devoted to present a detailed account of the asymptotic behavior as $t \rightarrow \infty$ of the solutions $u(x, t)$ of the equation

$$
u_{t}=\Delta\left(u^{m}\right)
$$

with exponent $m>1$. The study extends the well-known theory of the classical heat equation (HE, the case $m=1$ ) into a nonlinear situation, which needs a whole set of new tools. The space dimension can be any integer $N \geq 1$. They also present the extension of the results to exponents $m<1$ (fast-diffusion equation, FDE).

In this paper we study the problem

$$
\left\{\begin{array}{l}
u_{t}=\left(u^{m}\right)_{x x}, \quad x>0, t>0, \\
\left(u^{m}\right)_{x}(0, t)=u^{-\beta}(0, t), \quad t>0, \\
u(x, 0)=u_{0}(x), \quad x>0,
\end{array}\right.
$$

where $\beta>0, m>1, u_{0}$ is a smooth nonnegative function satisfying $\left(u_{0}^{m}\right)^{\prime}(x) \geq 0,\left(u_{0}^{m}\right)^{\prime \prime} \leq 0$ for $x>0$ and the compatibility conditions at $x=0$.

The study of quenching (in general the solution is defined up to $t=T$ but some term in the problem ceases to make sense) began with the work of Kawarada [4] appeared in 1975. In that paper he studied the semilinear heat equation as a singular reaction at level $u=1$. He proved that not only the reaction term, but also the time derivative blows up wherever $u$ reaches this value, see also [5]. Quenching problems have been studied by many authors, see [6-10] and the references 
therein.

The nonlinear parabolic equation

$$
u_{t}=\frac{m-1}{m}\left(u^{m}\right)_{x x}-(m-1) u^{m-p}\left|u_{x}\right|^{p}
$$

with $m \neq 0$ is a mathematical model for many physical problems corresponding to nonlinear diffusion with convection. The source term on the right-hand side of (1.2) is of convective nature. In the theory of un-natured porous medium equation, the convective part may represent the effect of gravity. Moreover, with $m=2$ is also a Boussinesq equation of hydrology, which is involved in various fields of petroleum technology and ground water hydrology. For instance, in [11], Zhang and Wang studied the following equation:

$$
\left\{\begin{array}{l}
u_{t}=\frac{m-1}{m}\left(u^{m}\right)_{x x}-(m-1) u^{m-p}\left|u_{x}\right|^{p} \\
-(m-1) u^{m-2}\left|u_{x}\right|^{2} \\
\left(u^{m}\right)_{x}(0, t)=0, \quad\left(u^{m}\right)_{x}(1, t)=m u^{q}(1, t), \\
u(x, 0)=u_{0}(x), \quad x \in[0,1]
\end{array}\right.
$$

for $(x, t) \in(0,1) \times(0, T)$, where $q \geq m>1$ and $p<2$ are parameters, and $u_{0}(x)>1$ is continuous and satisfies the compatibility conditions. They proved that, every solution of (1.3) will blow up in finite time for $q>m>1$ or for $q=m>1$ and $p<2$. And they got the blow up rate $u(x, t) \sim(T-t)^{-1 /(2 q-m-1)}$ for $q>m>1$ and $u(x, t) \sim(T-t)^{-1 /(m-1)}$ for $q=m>1$ and $p<2$.

The porous medium equation without convection has been considered extensively in the past few years. For instance, in [12], Galaktionov and Levine studied the following equation:

$$
\left\{\begin{array}{l}
u_{t}=\left(u^{m}\right)_{x x}, \quad(x, t) \in(0,+\infty) \times(0, T), \\
-\left(u^{m}\right)_{x}(0, t)=u^{q}(0, t), \quad t \in(0, T), \\
u(x, 0)=u_{0}(x), \quad x \in(0,+\infty) .
\end{array}\right.
$$

They proved that, if $0<q \leq(m+1) / 2$, then all nonnegative solutions to (1.4) were global; while for $q>(m+1) / 2$, the solutions to the equation would blow up in finite time. Moreover, if $(m+1) / 2<q \leq m+1$, all nonnegative solutions blow up in finite time; if $q>m+1$, global nontrivial nonnegative solutions existed.

Pablo, Quiros and Rossi [13] firstly distinguished nonsimultaneous quenching from simultaneous one. They considered a heat system coupled via inner absorptions,

$$
\left\{\begin{array}{l}
u_{t}=u_{x x}-v^{-p}, v_{t}=v_{x x}-u^{-q}, \\
u_{x}(0, t)=v_{x}(0, t)=u_{x}(1, t)=v_{x}(1, t)=0, \\
u(x, 0)=u_{0}(x), v(x, 0)=v_{0}(x), \quad x \in[0,1],
\end{array}\right.
$$

for $(x, t) \in(0,1) \times(0, T)$, where

$\min _{x \in[0,1]} u(x, t)=u(0, t), \min _{x \in[0,1]} v(x, t)=v(0, t)$

under certain assumptions on the initial data $u_{0}, v_{0}>0$. For the coupled equations (1.5), the following quenching rates were proved in [13]:

1) If quenching is non-simultaneous and, for instance, $v$ is the quenching component, then $v(0, t) \sim(T-t)$ for $t$ close to $T$.

2) If quenching is simultaneous, then for $t$ close to $T$,

a) $u(0, t) \sim(T-t)^{\frac{p-1}{p q-1}}, v(0, t) \sim(T-t)^{\frac{q-1}{p q-1}}$,

$p, q>1$ or $p, q<1$

b) $u(0, t), v(0, t) \sim(T-t)^{1 / 2}, p=q=1$;

c)

$$
\begin{aligned}
& u(0, t) \sim|\log (T-t)|^{-\frac{1}{q-1}}, \\
& v(0, t) \sim(T-t)|\log (T-t)|^{\frac{q}{q-1}}, q>p=1 .
\end{aligned}
$$

For the system

$$
\left\{\begin{array}{l}
u_{t}=u_{x x}, \quad v_{t}=v_{x x}, \quad(x, t) \in(0,1) \times(0, T), \\
u_{x}(0, t)=0, \quad u_{x}(1, t)=-v^{-p}(1, t), \quad t \in(0, T), \\
v_{x}(0, t)=0, \quad v_{x}(1, t)=-u^{-q}(1, t), \quad t \in(0, T), \\
u(x, 0)=u_{0}(x), v(x, 0)=v_{0}(x), \quad x \in[0,1],
\end{array}\right.
$$

the finite time quenching results with the coupled singular nonlinear boundary flux were obtained by Zheng and Song [14], other than the situation in the model of (1.5) with coupled nonlinear absorption terms. The quenching in (1.6) may be either simultaneous or non-simultaneous. This is determined by particular ranges of nonlinear exponents and the initial data. They showed that $\{x=1\}$ is the only quenching point and there are three kinds of simultaneous quenching rates can be briefly described in the following conclusions:

1) $u(1, t) \sim(T-t)^{\alpha / 2}, v(1, t) \sim(T-t)^{\beta / 2}$, $p, q>1$ or $p, q<1$;

2) $u(1, t) \sim(T-t)^{1 / 4}, v(1, t) \sim(T-t)^{1 / 4}, p=q=1$;

3)

$$
\begin{aligned}
& u(1, t) \sim|\log (T-t)|^{-\frac{1}{q-1},} \\
& v(1, t) \sim(T-t)|\log (T-t)|^{\frac{q}{q-1}}, 1=p<q,
\end{aligned}
$$

where $\alpha=(p-1) /(p q-1)$ and $\beta=(q-1) /(p q-1)$.

And $v(1, t) \sim(T-t)^{1 /(p+1)}$ for non-simultaneous quenching with $v$ quenching only.

In [15], Fila and Levine studied the quenching pro- 
blem for the scalar case

$$
\left\{\begin{array}{l}
u_{t}=u_{x x}, \quad(x, t) \in(0,1) \times(0, T) \\
u_{x}(0, t)=0, \quad u_{x}(1, t)=-u^{-q}(1, t), \quad t \in(0, T) \\
u(x, 0)=u_{0}(x)>0, \quad x \in[0,1]
\end{array}\right.
$$

and obtained that $u(1, t) \sim(T-t)^{\frac{1}{(2 q+1)}}$.

In [7], Deng and Xu studied the quenching problem

$$
\left\{\begin{array}{l}
(\psi(u))_{t}=u_{x x}, \quad 0<x<1, t>0 \\
u_{x}(0, t)=0, u_{x}(1, t)=-g(u(1, t)), \quad t>0 \\
u(x, 0)=u_{0}(x), \quad 0 \leq x \leq 1
\end{array}\right.
$$

For the special case $\psi(u)=u^{m}$ and $g(u)=u^{-\beta}$, here $0<m, \beta<\infty$, it is well known, $0<m<1$ corresponds to the porous medium case, $m>1$ refers to the fast diffusion case, and when $m=1$, the equation in (2.8) reduces to the heat equation. They obtained that $x$ $=1$ is the only quenching point and the quenching rate is $u(1, t) \sim(T-t)^{1 /(m+2 \beta+1)}$ for the porous medium case.

Our main purpose in this paper is to examine the quenching behavior of the solution of the problem (1.1), that is, the solution reaches zero in finite time and the quenching rate about $x$ and $t$. We get the same quenching rate as in [7]. Furthermore, we give the asymptotic profile $u(x, T) \sim x^{1 /(m+\beta)}$.

The paper is organized as follows: In Section 2, we prove that quenching occurs only at $x=0$. In Section 3 , we derive estimates for the quenching rate. In Section 4, we give the precise asymptotic profile near $x=0$.

\section{Quenching on the Boundary}

We state a lemma that guarantees that, for certain initial data, the solution of (1.1) decreases with $t$ and increases with $x$.

Lemma 2.1. Suppose that $\left(u_{0}^{m}\right)^{\prime}(x) \geq 0$ and $\left(u_{0}^{m}\right)^{\prime \prime}(x) \leq 0$ for $x>0$, then $\left(u^{m}\right)_{x}(x, t)>0$ and $u_{t}(x, t)<0$ in $(0, \infty) \times(0, T)$.

Proof. Let $v(x, t)=\left(u^{m}\right)_{x}(x, t)$ and $w(x, t)=u_{t}$. Then $v(x, t)$ and $w(x, t)$ satisfy

$$
\left\{\begin{array}{l}
v_{t}=m u^{m-1} v_{x x}+m(m-1) u^{m-2} u_{x} v_{x}, \\
x>0, t>0 \\
v(0, t)=\left(u^{m}\right)_{x}(0, t)=u^{-\beta}(0, t), \quad t>0, \\
v(x, 0)=\left(u_{0}^{m}\right)^{\prime}(x) \geq 0, \quad x>0,
\end{array}\right.
$$

and

$$
\left\{\begin{array}{l}
w_{t}=\left(m u^{m-1} w\right)_{x x}, \quad x>0, t>0, \\
\left(m u^{m-1} w\right)_{x}(0, t)=-\beta u^{-\beta-1}(0, t), \quad t>0, \\
w(x, 0)=\left(u_{0}^{m}\right)^{\prime \prime} \leq 0, \quad x>0,
\end{array}\right.
$$

respectively. From the maximum principle, it follows that $v>0, w<0$, and hence $\left(u^{m}\right),(x, t)>0$ and $u_{t}(x, t)<0$ in $(0, \infty) \times(0, T)$.

By the monotone of the $u(x, t)$ on $x$, we can find the quenching point in a finite interval $\left[0, x_{1}\right]$, where $1<x_{1}<K<\infty$. Let $u$ be a solution of (1.1) with $0<u_{0} \leq M$ on $\left[0, x_{1}\right]$. Then $0<u \leq M$ for all $t$ in the existence interval and $x \in\left[0, x_{1}\right]$. We now present the quenching result for the problem (1.1).

Theorem 2.1. Assume $\beta>0$. Then every solution $u$ of (1.1) quenches in finite time with the only quenching point $x=0$.

Proof. By Lemma 2.1, we know $\left(u^{m}\right)_{x}(x, t)>0$ and $u_{t}(x, t)<0$. Thus,

$$
\min _{x \in\left[0, x_{1}\right]} u(x, t)=u(0, t), \quad t \in[0, T) .
$$

For $F(t)=\int_{0}^{x_{1}} u(x, t) \mathrm{d} x$, we have

$$
\begin{aligned}
F^{\prime}(t) & =\int_{0}^{x_{1}} u_{t}(x, t) \mathrm{d} x=\int_{0}^{x_{1}}\left(u^{m}\right)_{x x} \mathrm{~d} x \\
& =\left(u^{m}\right)_{x}\left(x_{1}, t\right)-\left(u^{m}\right)_{x}(0, t) .
\end{aligned}
$$

Since $\left(u^{m}\right)_{x x}=u_{t}<0$, we have

$$
\left(u^{m}\right)_{x}\left(x_{1}, t\right)<\left(u^{m}\right)_{x}(0, t)
$$

There exist $\delta>0$ small enough such that

$$
\left(u^{m}\right)_{x}\left(x_{1}, t\right) \leq(1-\delta)\left(u^{m}\right)_{x}(0, t) \text {. }
$$

Thus

$$
\begin{aligned}
F^{\prime}(t) & =\left(u^{m}\right)_{x}\left(x_{1}, t\right)-\left(u^{m}\right)_{x}(0, t) \\
& \leq-\delta\left(u^{m}\right)_{x}(0, t) \\
& =-\delta u^{-\beta}(0, t) \leq-\delta M^{-\beta}
\end{aligned}
$$

and so

$$
u(0, t) \leq \frac{F(t)}{x_{1}} \leq \frac{F(0)}{x_{1}}-\frac{\delta M^{-\beta} t}{x_{1}} \leq M-\frac{\delta M^{-\beta} t}{x_{1}},
$$

which means that there exists $T>0$ such that $\lim _{t \rightarrow T^{-}} u(0, t)=0$.

To show that $x=0$ is the unique quenching point, it suffices to prove that the quenching cannot occur at any inner point $x_{0} \in(0,1 / 2)$. Define

$$
h(x, t)=\left(u^{m}\right)_{x}(x, t)-\frac{\varepsilon}{2 M^{\beta}} x^{2},
$$


where $\varepsilon>0$. Since $\left(u^{m}\right)_{x}(x, T / 2)>0$ for $x>0$, there exists $\varepsilon_{0}>0$ such that $\left(u^{m}\right)_{x}(x, T / 2) \geq \varepsilon_{0}>0$ for $x \in[0,3 / 4]$. If we take $\varepsilon \leq 32 M^{\beta} \varepsilon_{0} / 9$, then $h(x, T / 2) \geq 0, x \in[0,3 / 4]$. We have

$$
\left\{\begin{array}{l}
h_{t}-m u^{m-1} h_{x x}-m(m-1) u^{m-2} u_{x} h_{x} \\
=m u^{m-1} \frac{\varepsilon}{M^{\beta}}+m(m-1) u^{m-2} u_{x} \frac{\varepsilon}{M^{\beta}} x>0, \\
(x, t) \in(0,3 / 4) \times(T / 2, T), \\
h(0, t)=\left(u^{m}\right)_{x}(0, t)=u^{-\beta}(0, t)>0, \\
t \in(T / 2, T), \\
h(3 / 4, t)=\left(u^{m}\right)_{x}(3 / 4, t)-\frac{9 \varepsilon}{32 M^{\beta}} \geq \varepsilon_{0}-\varepsilon_{0}=0 \\
t \in(T / 2, T) .
\end{array}\right.
$$

By the maximum principle, $h \geq 0$ in $(0,3 / 4) \times(T / 2, T)$, which means that

$$
\begin{gathered}
\left(u^{m}\right)_{x}(x, t)-\frac{\varepsilon}{2 M^{\beta}} x^{2}=h \geq 0, \\
(x, t) \in(0,1 / 2) \times(T / 2, T) .
\end{gathered}
$$

Integrating with respect to $x$, we obtain

$$
\begin{gathered}
u^{m}(x, t) \geq u^{m}(0, t)+\frac{\varepsilon}{6 M^{\beta}} x^{3}, \\
(x, t) \in(0,1 / 2) \times(T / 2, T),
\end{gathered}
$$

and hence for any $x_{0} \in(0,1 / 2)$,

$$
\lim _{t \rightarrow T^{-}} \inf u\left(x_{0}, t\right) \geq\left(\frac{\varepsilon x_{0}^{3}}{6 M^{\beta}}\right)^{\frac{1}{m}}>0 .
$$

We have shown that quenching cannot occur in the interior of $\left(0, x_{1}\right)$. By the monotone, the proof is complete.

\section{Bounds for the Quenching Rate}

In this section, we establish bounds on the quenching rate.

Theorem 3.1. Suppose that $\left(u_{0}^{m}\right)^{\prime}(x) \geq 0$ and $\left(u_{0}^{m}\right)^{\prime \prime}(x) \leq 0$. Then the solution of (1.1) satisfies

$$
C_{1} \leq u(0, t)(T-t)^{-\frac{1}{m+2 \beta+1}} \leq C_{2},
$$

where $C_{1}$ and $C_{2}$ are positive constants.

Proof. We first present the upper bound. Let $\Phi(x, t)=\left(u^{m}\right)_{x}-u^{r}(x, t) u^{\eta}(x, t)$, where $r<0, \quad \eta>0$ and $r+\eta=-\beta$. We have

$$
\begin{aligned}
& \Phi_{t}-m u^{m-1} \Phi_{x x}-m(m-1) u^{m-2} u_{x} \Phi_{x} \\
& =-r u^{r-1} u_{t} u^{\eta}(0, t)-\eta u^{r} u^{\eta-1}(0, t) u_{t}(0, t) \\
& +m r(r-1) u^{m+r-3} u_{x}^{2} u^{\eta}(0, t)+m r u^{m+r-2} u_{x x} u^{\eta}(0, t) \\
& +m r(m-1) u^{m+r-3} u_{x}^{2} u^{\eta}(0, t) \\
& =-r u^{r-1} u^{\eta}(0, t)\left(u_{t}-m u^{m-1} u_{x x}(x, t)-m(m-1) u^{m-2} u_{x}^{2}\right) \\
& +m r(r-1) u^{m+r-3} u_{x}^{2} u^{\eta}(0, t)-\eta u^{r} u^{\eta-1}(0, t) u_{t}(0, t) \\
& =m r(r-1) u^{m+r-3} u_{x}^{2} u^{\eta}(0, t)-\eta u^{r} u^{\eta-1}(0, t) u_{t}(0, t) \\
& \geq 0
\end{aligned}
$$

for $x>0, t>0$, and

$$
\begin{aligned}
\Phi(0, t) & =\left(u^{m}\right)_{x}(0, t)-u^{r}(0, t) u^{\eta}(0, t) \\
& =u^{-\beta}(0, t)-u^{r+\eta}(0, t)=0 .
\end{aligned}
$$

By the maximum principle, we have

$$
\Phi(x, t)=\left(u^{m}\right)_{x}-u^{r}(x, t) u^{\eta}(0, t) \geq 0 .
$$

Then $\Phi_{x}(0, t) \geq 0$, i.e.,

$$
\left(u^{m}\right)_{x x}(0, t)-r u^{r-1}(0, t) u_{x}(0, t) u^{\eta}(0, t) \geq 0 .
$$

Hence

$$
u_{t}(0, t) \geq \frac{r}{m} u^{r-1+1-m-\beta+\eta}(0, t)=\frac{r}{m} u^{-m-2 \beta}(0, t) .
$$

Integrating the equality (3.1) from $t$ to $T$, we obtain

$$
u^{m+2 \beta+1}(0, t) \leq C_{2}(T-t) .
$$

Thus we prove the desired upper bound.

We then give the lower bound. We use a modification of an argument from [16]. For $t \in[\tau, T]$ with some $\tau$ such that $u(0, t)<1$, set

$$
y(t)=u^{\lambda}(0, t) \int_{0}^{\varepsilon(t)} u(x, t) \mathrm{d} x
$$

with

$$
\varepsilon(t)=u^{m+\beta}(0, t),
$$

where $\lambda>-(m+\beta)$. By $\left(u^{m}\right)_{x x}<0$, we have

$$
\left(u^{m}\right)_{x}(\varepsilon(t), t)<\left(u^{m}\right)_{x}(0, t) .
$$

There exists a $\eta>0$ small enough such that

$$
\left(u^{m}\right)_{x}(\varepsilon(t), t)<(1-\eta)\left(u^{m}\right)_{x}(0, t) .
$$

A routine calculation shows

$$
\begin{aligned}
y^{\prime}(t) & =\lambda u^{\lambda-1}(0, t) u_{t}(0, t) \int_{0}^{\varepsilon(t)} u(x, t) \mathrm{d} x \\
& +u^{\lambda}(0, t)\left(\left(u^{m}\right)_{x}(\varepsilon(t), t)-\left(u^{m}\right)(0, t)\right) \\
& +(m+\beta) u^{\lambda+m+\beta-1}(0, t) u(\varepsilon(t), t) \\
& \leq u^{\lambda-1}(0, t) u_{t}(0, t) I(t)-\eta u^{\lambda-\beta}(0, t) .
\end{aligned}
$$


Here

$$
\begin{aligned}
I(t) & =\lambda \int_{0}^{\varepsilon(t)} u(x, t) \mathrm{d} x \\
& +(m+\beta) u^{m+\beta}(0, t) u(\varepsilon(t), t) .
\end{aligned}
$$

Since $\left(u^{m}\right)_{x} \geq 0$ and $\left(u^{m}\right)_{x x} \leq 0$ in $[0, \infty) \times[\tau, T)$, we find

$$
u(0, t) \leq u(x, t) \leq u(\varepsilon(t), t) \leq 2 u(0, t)
$$

for any $x \in[0, \varepsilon(t)]$ and $t \in[\tau, T)$. By (3.2), (3.3) and (3.4), we have

$$
u^{\lambda+m+\beta+1}(0, t) \leq y(t) \leq 2 u^{\lambda+m+\beta+1}(0, t)
$$

or equivalently,

$$
\begin{aligned}
u(0, t) \leq & y^{1 /(\lambda+m+\beta+1)}(t) \leq C_{3} u(0, t) \text { for } t \in[\tau, T) \\
I(t) & =(\lambda+m+\beta) \int_{0}^{\varepsilon(t)} u(x, t) \mathrm{d} x \\
& \quad(m+\beta) \int_{0}^{\varepsilon(t)}(u(x, t)-u(\varepsilon(t), t)) \mathrm{d} x \\
= & (\lambda+m+\beta) \int_{0}^{\varepsilon(t)} u(x, t) \mathrm{d} x \\
\quad & -(m+\beta) \int_{0}^{\varepsilon(t)} u_{x}(\xi(t), t)(x-\varepsilon(t)) \mathrm{d} x \\
\geq & (m+\lambda+\beta) u^{m+\beta+1}(0, t) \\
\quad & \quad \frac{m+\beta}{2} u^{2 m+2 \beta}(0, t) u_{x}(\xi(t), t) \\
\geq 0, \quad &
\end{aligned}
$$

where $0<\xi(t)<\varepsilon(t), u_{x}>0$ and $\int_{0}^{\varepsilon(t)}(x-\varepsilon(t)) \mathrm{d} x=-\varepsilon^{2}(t) / 2$. Then it follows that

$$
y^{\prime}(t) \leq-\eta u^{\lambda-\beta}(0, t) \leq-C_{4} y^{\frac{\lambda-\beta}{\lambda+m+\beta+1}}(t) .
$$

Integrating the above equality from $t$ to $T$, we obtain

$$
y^{\frac{2 \beta+m+1}{\lambda+m+\beta+1}}(t) \geq C_{5}(T-t) \text {. }
$$

That is,

$$
y^{\frac{1}{m+\lambda+\beta+1}} \geq C_{1}(T-t)^{2 \beta+m+1},
$$

which in conjunction with (3.5) yields the desired lower bound.

\section{Asymptotic Profile}

In this section, we shall derive the following precise asymptotic profile near $x=0$.

Theorem 4.1. Suppose that $u$ is the solution of (1.1) and assume that the quenching occurs at a finite time $t=T$, then there exist $c_{1}, c_{2}>0$ such that

$$
c_{1} x^{1 /(m+\beta)} \leq u(x, t) \leq c_{2} x^{1 /(m+\beta)}, x \geq 0,0<T-t \ll 1 .
$$

We first prove a lemma as follows.

Lemma 4.1. Assume that $u$ is the solution of (1.1) and assume that the quenching occurs at a finite time $t=T$, then there exists $a \quad c_{3}>0$ such that

$$
x u_{x}(x, t) \geq c_{3} u(x, t), \quad x \geq 0,0<T-t \ll 1 .
$$

Proof. Let $J=x^{2}\left(u^{m}\right)_{x}(x, t)-c_{4} x u^{m}(x, t)$, where $c_{4}>\frac{2 m}{m-1}$. Then $J$ satisfies

$$
\begin{aligned}
J_{t}- & m u^{m-1} J_{x x}-m(m-1) u^{m-2} u_{x} J_{x} \\
= & -c_{4} m x u^{m-1} u_{t}-m\left(4-c_{4}\right) x u^{m-1}\left(u^{m}\right)_{x x} \\
& -m(m-1)\left(2-c_{4}\right) x u^{m-2}\left(u^{m}\right)_{x} u_{x} \\
& +c_{4} m(m-1) u^{2 m-2} u_{x}-m\left(2-2 c_{4}\right) u^{m-1}\left(u^{m}\right)_{x} \\
= & -4 m x u^{m-1}\left(u^{m}\right)_{x x}+c_{4} m(m-1) u^{2 m-2} u_{x} \\
& \quad-m(m-1)\left(2-c_{4}\right) x u^{m-2}\left(u^{m}\right)_{x} u_{x} \\
& +c_{4} m(m-1) u^{2 m-2} u_{x} \\
\geq & 0,
\end{aligned}
$$

and $J(0, t)=0$. Then

$$
x^{2}\left(u^{m}\right)_{x}(x, t) \geq c_{4} x u^{m}(x, t),
$$

and the lemma is proved, where $c_{3}>\frac{2}{m-1}$.

Proof of Theorem 4.1. We first present the lower bound. Let

$$
J:=\left(u^{m}\right)_{x}(x, t)-u^{-\beta}(x, t) .
$$

Then $J$ satisfies

$$
\begin{aligned}
& J_{t}-m u^{m-1} J_{x x}-m(m-1) u^{m-2} u_{x} J_{x} \\
& =m \beta(1+\beta) u^{m-\beta-3}\left(u_{x}\right)^{2}>0,
\end{aligned}
$$

and

$$
J(0, t)=\left(u^{m}\right)_{x}(0, t)-u^{-\beta}(0, t)=0 .
$$

By the maximum principle, we obtain

$$
J=\left(u^{m}\right)_{x}(x, t)-u^{-\beta}(x, t) \geq 0 .
$$

Then

$$
u^{m+\beta-1} u_{x} \geq 1 / m .
$$

Integrating the above equality from 0 to $x$, we obtain that

$$
u(x, t) \geq c_{1} x^{1 / m+\beta}
$$

We then give the upper bound. Let

$$
\Phi=x^{\alpha}\left(u^{m}\right)_{x}-c_{5} u^{\lambda}
$$


where $0<\alpha=\frac{2 \beta+1}{2(m+\beta)}<1, \lambda=\frac{1}{2}$ and $c_{5}>0$.

$\Phi_{t}-m u^{m-1} \Phi_{x x}-m(m-1) u^{m-2} u_{x} \Phi_{x}+2 m \alpha x^{-1} u^{m-1} \Phi_{x}$

$=-c_{5} \lambda u^{\lambda-1} u_{t}-m \alpha(\alpha-1) x^{\alpha-2} u^{m-1}\left(u^{m}\right)_{x}$

$+c_{5} m \lambda(\lambda-1) u^{m+\lambda-3}\left(u_{x}\right)^{2}+c_{5} m \lambda u^{m+\lambda-2} u_{x x}$

$-\alpha m(m-1) u^{m-2} x^{\alpha-1} u_{x}\left(u^{m}\right)_{x}$

$+c_{5} m \lambda(m-1) u^{m+\lambda-3}\left(u_{x}\right)^{2}$

$+2 m \alpha^{2} x^{\alpha-2}\left(u^{m}\right)_{x} u^{m-1}-2 c_{5} m \alpha \lambda u^{m+\lambda-2} x^{-1} u_{x}$

$=-m \alpha(\alpha-1) x^{\alpha-2} u^{m-1}\left(u^{m}\right)_{x}+c_{5} m \lambda(\lambda-1) u^{m+\lambda-3}\left(u_{x}\right)^{2}$

$-\alpha m(m-1) u^{m-2} x^{\alpha-1} u_{x}\left(u^{m}\right)_{x}+2 m \alpha^{2} x^{\alpha-2}\left(u^{m}\right)_{x} u^{m-1}$

$-2 c_{5} m \alpha \lambda u^{m+\lambda-2} x^{-1} u_{x}$

$=m \alpha(1+\alpha) x^{\alpha-2} u^{m-1}\left(u^{m}\right)_{x}$

$-\alpha m(m-1) u^{m-2} x^{\alpha-1} u_{x}\left(u^{m}\right)_{x}$

$+c_{5} m \lambda(\lambda-1) u^{m+\lambda-3}\left(u_{x}\right)^{2}-2 c_{5} m \alpha \lambda u^{m+\lambda-2} x^{-1} u_{x}$

$=m \alpha x^{\alpha-2} u^{m-2}\left(u^{m}\right)_{x}\left[(1+\alpha) u-(m-1) x u_{x}\right]$

$+c_{5} m \lambda(\lambda-1) u^{m+\lambda-3} u_{x}^{2}-2 c_{5} m \alpha \lambda u^{m+\lambda-2} x^{-1} u_{x}$

$\leq m \alpha x^{\alpha-2} u^{m-1}\left(u^{m}\right)_{x}\left[1+\alpha-c_{3}(m-1)\right]$

$+c_{5} m \lambda(\lambda-1) u^{m+\lambda-3} u_{x}^{2}-2 c_{5} m \alpha \lambda u^{m+\lambda-2} x^{-1} u_{x}$

$\leq 0$.

On the other hand,

$$
\Phi(0, t)=-c_{5} u^{\lambda}(0, t)<0 .
$$

By the maximum principle, we have

$$
\Phi=x^{\alpha}\left(u^{m}\right)_{x}-c_{5} u^{\lambda} \leq 0 .
$$

Then

$$
u^{m-1-\lambda} u_{x} \leq c_{5} x^{-\alpha}
$$

Integrating the above equality, we obtain

$$
u(x, t) \leq c_{2} x^{\frac{1-\alpha}{m-\lambda}}=c_{2} x^{1 /(m+\beta)} .
$$

Remark 4.1. Let $t \rightarrow T$, we can get

$$
c_{1} x^{1 /(m+\beta)} \leq u(x, T) \leq c_{2} x^{1 /(m+\beta)}, \quad x \geq 0 .
$$

\section{References}

[1] L. S. Leibenzon, "The Motion of a Gas in a Porous Medium," Russian Academy of Sciences, Moscow, 1930.

[2] M. Muskat, "The Flow of Homogeneous Fluids through Porous Media,” McGraw-Hill, New York, 1937.

[3] J. L. Vázquez, "Asymptotic Behaviour for the Porous
Medium Equation Posed in the Whole Space," Journal of Evolution Equations, Vol. 3, No. 1, 2003, pp. 67-118. doi:10.1007/s000280300004

[4] H. Kawarada, "On Solutions of Initial Boundary Value Problem for $u_{t}=u_{x x}=1 /(1-u)$," Publications of the Research Institute for Mathematical Sciences, Vol. 10, 1975, pp. 729-736. doi:10.2977/prims/1195191889

[5] C. Y. Chan and M. K. Kwong, "Quenching Phenomena for Singular Nonlinear Parabolic Equations," Nonlinear Analysis, Vol. 12, No. 2, 1998, pp. 1377-1383. doi:10.1016/0362-546X(88)90085-5

[6] C. Y. Chan, "New Results in Quenching," Proceeding of 1 st World Congress of Nonlinear Analysts, Tampa, Vol. 1, 19-26 August 1992, pp. 427-434.

[7] K. Deng and M. X. Xu, "Quenching for a Nonlinear Diffusion Equation with a Singular Boundary Condition," Zeitschrift fur Angewandte Mathematik und Physik, Vol. 50, No. 4, 1999, pp. 574-584. doi:10.1007/s000330050167

[8] K. Deng and M. X. Xu, "On Solutions of a Singular Diffusion Equation," Nonlinear Analysis, Vol. 41, No. 3-4, 2000, pp. 489-500. doi:10.1016/S0362-546X(98)00292-2

[9] H. A. Levine, “Advances in Quenching," Proceeding of International Conference on Reaction-Diffusion Equations and Their Equilibrium States, Vol. 7, 1992, pp. 319-346.

[10] H. A. Levine and G. M. Lieberman, "Quenching of Solutions of Parabolic Equations with Nonlinear Boundary Conditions in Several Dimensions," Journal für Die Reine und Angewandte Mathematik, Vol. 1983, No. 345, 1983, pp. 23-38.

[11] Z. C. Zhang and B. Wang, "Blow-up Rate Estimate Parabolic Equation with Nonlinear Gradient Term," Applied Mathematics and Mechanics, Vol. 31, No. 6, 2010, pp. 787-796. doi:10.1007/s10483-010-1313-6

[12] V. A. Galaktionov and H. A. Levine, "On Critical Fujita Exponents for Heat Equations with Nonlinear Flux Conditions on the Boundary," Israel Journal of Mathematics, Vol. 94, No. 1, 1996, pp. 125-146.

[13] A. De Pablo, F. Quiros and J. D. Rossi, "Nonsimultaneous Quenching," Applied Mathematics Letters, Vol. 15, No. 3, 2002, pp. 265-269. doi:10.1016/S0893-9659(01)00128-8

[14] S. N. Zheng and X. F. Song, "Quenching Rates for the Heat Equatons with Coupled Singular Nonlinear Boundary Flux," Science in China Series A-Mathematics, Vol. 51, No. 9, 2008, pp. 1631-1643.

[15] M. Fila and H. A. Levine, "Quenching on the Boundary," Nonlinear Analysis, Vol. 21, No. 10, 1993, pp. 795-802. doi:10.1016/0362-546X(93)90124-B

[16] J. Filo, "Difusivity versus Absorption through the Boundary," Journal of Differential Equations, Vol. 99, No. 2, 1992, pp. 281-305. 